\title{
Investigation of $\mathrm{KTiOPO}_{4}$ nanocrystals by means of second-harmonic light emission
}

\author{
Chunyuan Zhou, Loc Le Xuan, Abdallah Slablab, Nicolas Sandeau, \\ Sophie Brasselet, Dominique Chauvat, and Jean-François Roch \\ Laboratoire de Photonique Quantique et Moléculaire, ENS Cachan, UMR CNRS 8537, \\ 61 avenue du Président Wilson, 94235 Cachan Cedex, France
}

Received August 28, 2007

\begin{abstract}
The finding of nonlinear nanometric-sized probes is of key importance for the development of nonlinear microscopy in physical as well as biological sciences. We isolate nonlinear $\mathrm{KTiOPO}_{4}$ nanocrystals and study them by second-harmonic generation microscopy (SHGM) and atomic force microscopy (AFM) independently. With both polarization analysis and defocused imaging of the emitted second harmonic field, we extract the Euler angles of the crystalline axes of a single nanocrystal. A balanced coherent optical homodyne detection shows the coherent nature of the nanocrystal nonlinear emission and allows a phase measurement of the emitted second-harmonic field. These features make the $\mathrm{KTiOPO}_{4}$ nanocrystal a good candidate for a vectorial probe of electromagnetic near fields.
\end{abstract}

OCIS codes: $190.3970,170.5810,160.4330$.

Nonlinear multiphoton microscopies are powerful and versatile methods to study physical surfaces ${ }^{[1]}$, molecular monolayers ${ }^{[2,3]}$, and biological membranes ${ }^{[4,5]}$. In this context, the studies of single nanoobjects, either resulting from crystalline growth ${ }^{[6-9]}$ or artificially designed with focused ion beams such as a nonlinear antenna ${ }^{[10]}$, have recently been reported. Nonlinear emissions then serve to reveal the symmetry of the nanoobject ${ }^{[11]}$ and the order of underlying light-matter interactions ${ }^{[10]}$. Standard nano-emitters such as ensemble of ordered asymmetric molecules, quantum dots or metallic beads, show interesting nonlinear properties, which have already been applied to biology ${ }^{[4,12]}$, but they are not ideal in every respect. Under intense illuminating light, molecular systems photobleach ${ }^{[13]}$, quantum dots can be photostable but strongly blink, and metallic nanospheres have a response which is highly dependent on the exact shape of the nanoparticle surface ${ }^{[14]}$. Other possible candidates are $\mathrm{ZnO}^{[8]}$ or $\mathrm{GaAs}^{[9]}$ cylindrical nanowires. However, in the reported studies, while they have nanometric transverse sections, they exhibit micrometric length along their $c$-axis. Therefore, there is a strong incentive to find new nanometric-sized nonlinear nano-objects, which emit a stable blinking-free optical field under intense focused laser beams. Here we investigate the properties of single $\mathrm{KTiOPO}_{4}$ nanocrystals in second-harmonic generation microscopy (SHGM). Using polarization analysis and defocused imaging of the second-harmonic emitted field, the Euler angles of the crystalline axes of a nanocrystal are measured. Furthermore, we have added a balanced coherent homodyne detection to the second-harmonic microscope, which proves the coherent nature of the interaction and allows us to detect isolated nano-objects with high signal-to-noise ratio and phase sensitivity.

Potassium titanyl phosphate $\left(\mathrm{KTiOPO}_{4}, \mathrm{KTP}\right)$ is well known to constitute an excellent crystal for nonlinear optics and is used to design frequency doublers or converters. This electric polar crystal in the noncentrosymmetric Pna2 $2_{1}$ space group shows large nonlin- ear coefficients, of the order of $17 \mathrm{pm} / \mathrm{V}$, when excited in the near infrared ${ }^{[15]}$. Large monocrystals can be grown by flux methods around $900{ }^{\circ} \mathrm{C}^{[16]}$. In order to obtain nanometric-sized crystals of KTP, we get the powder which remains in the trough at the end of the flux process of a large crystal. The KTP powder is provided by D. Lupinski and Ph. Villeval with Crystal Laser S. A., Nancy, France. Then the KTP powder is diluted in isopropanol and mixed with polyvynilpyrolidone (PVP). A centrifugation method ${ }^{[17]}$ is applied to obtain a colloidal solution with nanoparticles of average size $150 \mathrm{~nm}$, but containing also much smaller ones as shown below.

Since we are interested in studying well-isolated nanocrystals, samples are prepared to enable finding out the same single nano-object under an atomic force microscope (AFM) and a home-made SHGM (described in Fig. 1). The colloidal solution is spin-coated on a glass cover slip, which was previously cleaned with oxygen plasma, and results in a uniform 100-nm thin polymer coating embedding nanocrystals. By using the technique of reactive ion etching, we remove the covering polymer while letting the nano KTPs rest on the cover slip, which makes it possible to observe the profiles of the nanocrystals by AFM scan.

To reveal the presence of nonlinear emitters, a SHGM scan is performed. Laser pulses of $100 \mathrm{fs}$ emitted by a mode-locked Ti:sapphire laser at $86-\mathrm{MHz}$ repetition rate are tightly focused on the sample with a high numerical aperture $(\mathrm{NA}=1.4), 100 \times$ microscope objective leading to an $\sim 350 \mathrm{~nm}$ full-width at half-maximum (FWHM) diameter focal spot. We choose the excitation wavelength at $986 \mathrm{~nm}$ so that the light-matter interaction is highly non-resonant both at this fundamental and the corresponding $493 \mathrm{~nm}$ second-harmonic wavelengths, where KTP is still highly transparent. The sample is mounted on an $x-y$ translation stage which allows a raster scan with $\sim 30 \mathrm{~nm}$ resolution. Whenever a KTP nanocrystal generates second-harmonic photons, they are collected back through the same objective and 


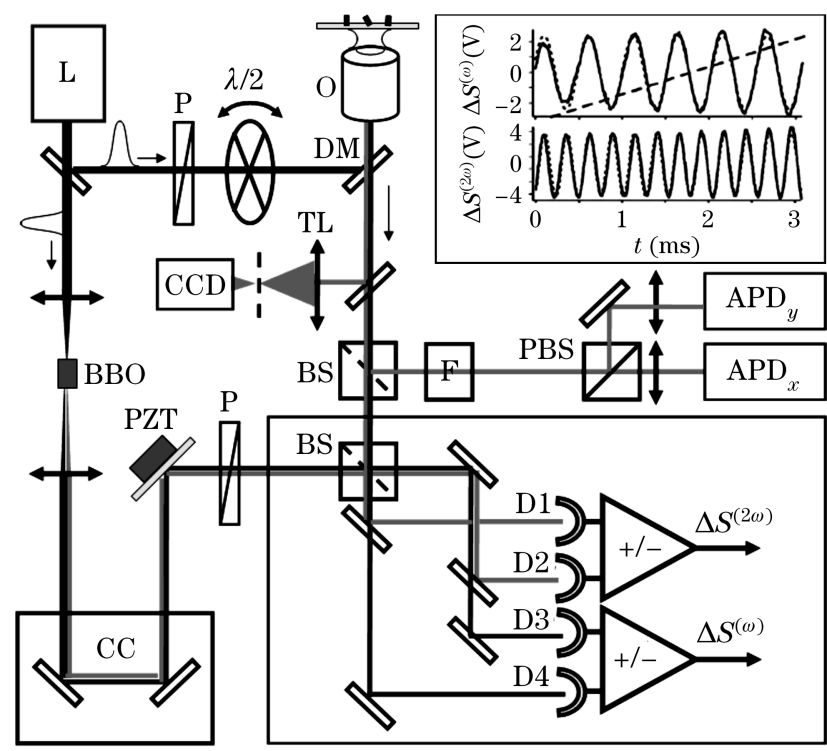

Fig. 1. Experimental set-up for second-harmonic microscopy. L: femtosecond laser; BBO: nonlinear $\chi^{(2)}$ crystal; P: polarizer; O: microscope objective; BS: nonpolarizing beam splitter; CC: corner cube; PZT: mirror mounted on a piezoelectric transducer; F: SHG filter; $\lambda / 2$ : half-wave plate; DM: dichroic mirror; PBS: polarizing beam splitter; TL: tube lens; $\mathrm{APD}_{x}$, $\mathrm{APD}_{y}$ : avalanche photodiodes in photon counting regime; D1 and D2: p-i-n Si photodetectors of the balanced receiver, recording SHG at $2 \omega$ (a SHG filter, not shown, is put in front of the detectors); D3 and D4: the same for the balanced receiver at fundamental optical frequency (an IR filter, not shown, is put in front of the detectors). Inset: full lines, detected signals as a function of time for a forward translation of the mirror. Up: signal $\Delta S^{(\omega)}$ at fundamental frequency with a sinusoidal fit at frequency $f=1.9 \mathrm{kHz}$, and bottom: $\Delta S^{(2 \omega)}$ at SHG frequency with a sinusoidal fit at frequency $2 f=3.8 \mathrm{kHz}$. Dashed line: voltage applied to the $\mathrm{PZT}$ on half a period (vertical scale: a.u.).

transmitted through a dichroic mirror and an additional second-harmonic generation (SHG) filter centered at $(500 \pm 20) \mathrm{nm}$, which efficiently cut the reflected fundamental beam. Finally the SHG photons are detected by avalanche photodiodes in photon counting regime. Scanning the sample, for instance the area of Fig. 2(b), a SHG image is obtained. Nearly all these emitters can also be found unambiguously in the AFM image (see white circles in Fig. 2(a)), which gives the height of the nanocrystals. This height is taken as a measurement of the nanocrystal size. Since it is still possible that some polymer is left under a nanocrystal after the etching process, the measured size is an overestimation of the actual size. We also note that a lot of nano-objects observed in the AFM image actually do not emit. They are mostly polymer residue. Figures 2(c) and (d) show the AFM and SHG close scans of nano KTP \#1 respectively. The zoom of the AFM image shown in Fig. 2(c) reveals a single nanocrystal with a $60-\mathrm{nm}$ height and nanometric sizes in transverse dimensions. We note that the transverse size is certainly over-estimated, due to convolution of the nanocrystal shape with the AFM tip and to residual polymer that might have stuck to the nano-KTP sides. The corresponding zoom on the SHG image in Fig. 2(d) gives a diffraction limited spot with FWHM about $350 \mathrm{~nm}$, as expected for an emitter size well below
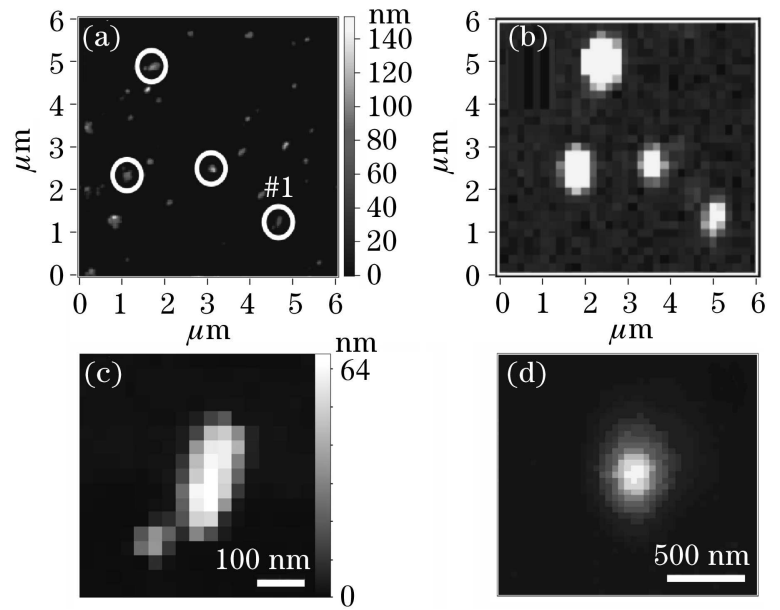

Fig. 2. Correspondence of nanoparticles in images taken with AFM and SHG. (a) Overview in AFM scan with circles pinpointing SHG emitting spots; (b) corresponding raster-scan SHG image; (c) close scan of AFM image; (d) SHG close scan.

the diffraction limit. The number of emitted photons at the center of the spot is large, about $3.5 \times 10^{3}$ photons per second for an incident power of $15 \mathrm{~mW}$, and a signalto-background ratio of 120 is measured.

Before analyzing experimentally the SHG emission from a single nonlinear emitter, we discuss it from a theoretical point of view. While an exact approach is possible ${ }^{[18]}$, we choose to refer to a simplified model ${ }^{[11]}$. We assume that the use of 2 nd-order susceptibility tensor $\chi^{(2)}$ of the bulk material is also valid at the nanoparticle level. It appears to be sufficient to explain the main features of our experimental results. If the tensor of the nanocrystal in its crystallographic axes is noted $\chi_{I, J, K}^{(2)}$, where $I, J, K$ are for $X, Y, Z$; the same tensor in the laboratory axes $\chi_{i, j, k}^{(2)}$, where $i, j$, $k$ are for $x, y, z$, is obtained by simple rotations, $\chi_{i, j, k}^{(2)}=\sum \chi_{I, J, K}^{(2)} \cos (i, I) \cos (j, J) \cos (k, K)$. For an incident electric field $\vec{E}^{(\omega)}$, the $i$ th component of the secondharmonic generated dipole is

$$
P_{i}^{(2 \omega)}=\sum_{j, k} \chi_{i, j, k}^{(2)}(-2 \omega ; \omega, \omega) E_{j}^{(\omega)} E_{k}^{(\omega)} .
$$

This resulting nonlinear dipole $\vec{P}^{(2 \omega)}$ radiates a second-harmonic field. In a given propagation direction $\vec{k}$, the far-field emission reads ${ }^{[19]}$

$$
\vec{E}_{\mathrm{FF}}^{(2 \omega)} \propto \vec{k} \times \vec{k} \times \vec{P}^{(2 \omega)} .
$$

Finally the intensity radiation diagram, $I_{i}^{(2 \omega)}(k) \propto$ $\left|E_{i, \mathrm{FF}}^{(2 \omega)}(k)\right|^{2}$, where $i=x, y$, is measured directly as a function of $\vec{k}$, or detected by focusing the emitted light on a detector leading to a signal proportional to $\left\langle I_{i}^{(2 \omega)}\right\rangle_{\Omega} \propto\left|\iint_{\Omega} E_{i, \mathrm{FF}}^{(2 \omega)} \mathrm{d} \Omega\right|^{2}$, where $\Omega$ is the detection solid angle. An advantage of the emission of nanocrystals much smaller than the wavelength with respect to macroscopic nonlinear crystals, is the near absence of phase shift between the second harmonic fields generated by different parts of the nanoparticle, leading to a 
constructive interference. Therefore phase-matching is avoided and any wavelength can be used to excite the nanocrystal. Another salient feature of the SHG of a nanocrystal as compared to other nanometric probes is the instrinsic sensitivity not only to the excitation power, but to the vectorial nature of the exciting field since KTP tensor $\chi_{i j k}^{(2)}$ includes 5 different non-null coefficients.

Figure 3 shows the experimental analysis for 4 nano KTPs. Since from Eq. (1) the SHG field is expected to be highly polarized, we decompose the SHG field on its $x$ and $y$ components with a polarizing beam splitter (PBS) cube and two avalanche photodiodes in photon counting regime. In order to further probe the nature of the dipolar emission as well as the symmetry of the crystal, the linearly polarized excitation field is rotated with a half-wave plate in the path of the incident beam (shown in Fig. 1). The resulting polar graphs displayed in Fig. 3 are typical dipolar emission patterns. Together with the knowledge of the whole set of nonlinear coefficients of $\mathrm{KTP}^{[15]}$ and Eq. (1), it allows to infer the projection of the radiating SHG dipole $\vec{P}^{(2 \omega)}$ on the $(x, y)$ plane of the sample, as well as the projection of the nanocrystal $c$-axis on this plane, i.e. Euler angle $\varphi$.

The polarization analysis technique has however a low sensitivity to any dipole component along the symmetry axis of the microscope, i.e., the $z$-axis. To retrieve the orientation of the dipole in three-dimension, we use the recently developed defocused imaging technique ${ }^{[20,21]}$, here generalized to the nonlinear case ${ }^{[22]}$. It is based on imaging the radiation pattern of the emitter onto a sensitive charge-coupled device (CCD) camera by positioning the camera slightly out of the focal plane of the tube lens. The image is then the product of a defocused Airy function and the radiation pattern of the dipole. In particular, this image is able to reveal any $z$-component in the emitting dipole. A specific feature of nonlinear defocused imaging is a non-uniform rotation of the defocused image as a function of the rotation of the linearly polarized excitation field. This feature is clearly expected from Eq. (1) since $\vec{P}^{(2 \omega)}$ orientation strongly depends on $\vec{E}^{(\omega)}$. It is then possible to extract the full set of Euler angles of the nanocrystals with high accuracy $^{[22]}$. Figure 3 shows the defocused images for an incident excitation field polarized along $x$ and the deduced three-dimensional orientations for all the four nonlinear emitters.

Finally, we test the coherent nature of the nonlinear emission of KTP nanocrystal using an interferometric

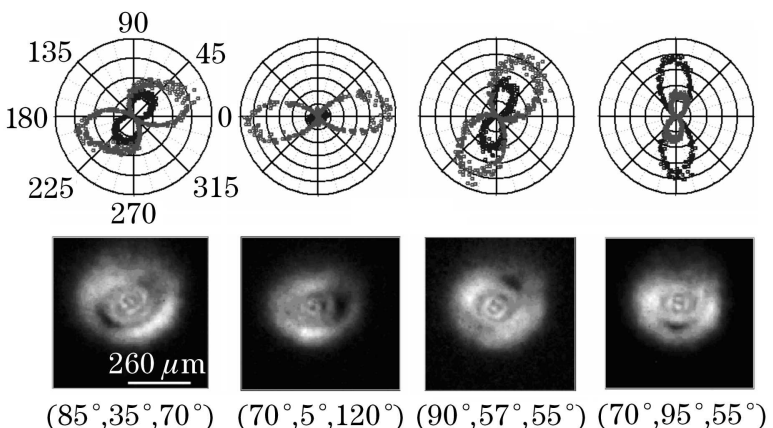

Fig. 3. Examples of some isolated nanocrystals. Euler angles $(\theta, \varphi, \Psi)$ for the different nanocrystals are given in the figures. detection method. A coherent detection of SHG has a few advantages such as SHG detection on a dark background, since incoherent emission from two-photon fluorescence would lead to no signal for instance, and SHG phase sensitivity, which is related to the absolute orientation of a nonlinear nanocrystal. The SHG field is associated with an electric field $E^{(2 \omega)}=\left|E^{(2 \omega)}\right| \exp \left[i \phi_{\mathrm{obj}}^{(2 \omega)}\right]$, where $\phi_{\mathrm{obj}}^{(2 \omega)}$ is the phase shift of the SHG field emitted by the nanocrystal as compared to the one of the incident fundamental field $E_{\text {in }}^{(\omega)}$. For a nonlinear crystal, the SHG field is $E^{(2 \omega)} \propto \chi^{(2)} E_{\mathrm{in}}^{(\omega) 2}$. Thus, $\phi_{\mathrm{obj}}^{(2 \omega)}$ reflects the phase of the nonlinear susceptibility $\chi^{(2)}$ which depends on the absolute orientation of the nonlinear response in respect to the crystal axis. Therefore determination of $\phi_{\mathrm{obj}}^{(2 \omega)}$ allows to infer the absolute orientation of the nonlinear crystal. In practice, the coherent phase measurement (shown at bottom-right part in Fig. 1), is realized by a balanced homodyne coherent detection ${ }^{[23]}$ where the emitted SHG field of a nanocrystal is mixed with an intense SHG coherent reference field or local oscillator with a nonpolarizing beam splitter (BS) cube. Each beam, signal and local oscillator, follows one path of a Mach-Zehnder interferometer. The lengths of the two arms of the interferometer are equalized, and chromatic dispersion is compensated with glass plates (not shown). Detecting and substracting the two output ports of the cube allows to extract only the optical interference term between the local oscillator and the small SHG field. Then, if the optical length of one path is modulated with a mirror mounted on a piezoelectric transducer (PZT), interference fringes are observed (inset in Fig. 1). This is a direct proof of the coherent nature of the nanocrystal nonlinear emission. The absolute orientation of a nanocrystal could then be determined, which provided one having a reference SHG active object with a known absolute orientation.

In summary, we have investigated the SHG properties of well-isolated nanometric sized KTP crystals and measured their sizes by AFM. In particular, the threedimensional orientation of these nanocrystals has been retrieved. We have shown the interferometric detection of SHG from these nanocrystals, which proves the coherent nature of the emission and paves the way to absolute orientation detection of an asymmetric SHG nanoemitter. A direct chemical synthesis of large density of nanocrystals with controlled monodisperse size distribution is also under way for potential physical or biological applications. We think that nano KTP adds new features to the toolbox of nonlinear microscopy, and the method presented here can be applied to other nonlinear nanoemitter candidates.

This work was supported by the AC Nanoscience Research Program and by Institut Universitaire de France. We are grateful to J. Lautru, N. Brosseau, C. Tard, S. Perruchas, T. Gacoin, F. Treussart, J.-P. Boilot, and J.-J. Greffet for their help. C. Zhou's e-mail address is czhou@lpqm.ens-cachan.fr.

\section{References}

1. J. Chen, S. Machida, and Y. Yamanoto, Opt. Lett. 23, 676 (1998). 
2. Y. R. Shen, Nature 337, 519 (1989).

3. K. Kemnitz, K. Bhattacharyya, J. M. Hicks, G. R. Pinto, B. Eisenthal, and T. F. Heinz, Chem. Phys. Lett. 131, 285 (1986).

4. L. Moreaux, O. Sandre, and J. Mertz, J. Opt. Soc. Am. B 17, 1685 (2000).

5. W. R. Zipfel, R. M. Williams, and W. W. Webb, Nature Biotechnology 21, 1369 (2003).

6. P. Alivisatos, Nature Biotechnology 22, 47 (2004).

7. F. Treussart, E. Botzung-Appert, N. T. Ha-Duong, A. Ibanez. J. F. Roch, and R. Pansu, Chem. Phys. Chem. 4, 757 (2003).

8. J. C. Johnson, H. Yan, R. D. Schaller, P. B. Petersen, P. D. Yang, and R. J. Saykally, Nano Lett. 2, 279 (2002).

9. J. P. Long, B. S. Simpkins, D. J. Rowenhorst, and P. E. Pehrsson, Nano Lett. 7, 831 (2007).

10. P. Mühlschlegel, H.-J. Eisler, O. J. F. Martin, B. Hecht, and D. W. Pohl, Science 308, 1607 (2005).

11. S. Brasselet, V. Le Floc'h, F. Treussart, J.-F. Roch, and J. Zyss, Phys. Rev. Lett. 92, 207401 (2004).

12. D. R. Larson, W. R. Zipfel, R. M. Williams, S. W. Clark, M. P. Bruchez, F. W. Wise, and W. W. Webb, Science 300, 1434 (2003).

13. G. H. Patterson and D. W. Piston, Biophys. J. 78, 2159
(2000).

14. D. Boyer, P. Tamarat, A. Maali, B. Lounis, and M. Orrit, Science 297, 1160 (2002).

15. R. L. Sutherland, Handbook of Nonlinear Optics (Dekker, New York, 1996).

16. I. Tordjman, R. Masse, and J. C. Guitel, Z. Kristallogr. 139, 100 (1974).

17. F. Treussart, V. Jacques, E. Wu, T. Gacoin, P. Grangier, and J. F. Roch, Physica B 376, 926 (2006).

18. J. I. Dadap, J. Shan, and T. F. Heinz, J. Opt. Soc. Am. B 21, 1328 (2004).

19. J. D. Jackson, Classical Electrodynamics (John Wiley \& Sons, New York, 1962).

20. M. Böhmer and J. Enderlein, J. Opt. Soc. Am. B 20, 554 (2003).

21. X. Brokmann, J.-P. Hermier, G. Messin, P. Desbiolles, J.-P. Bouchaud, and M. Dahan, Phys. Rev. Lett. 90, 120601 (2003).

22. N. Sandeau, L. Le Xuan, D. Chauvat, C. Zhou, J. F. Roch, and S. Brasselet, Opt. Express 15, 16051 (2007).

23. L. Le Xuan, S. Brasselet, F. Treussart, J.-F. Roch, F. Marquier, D. Chauvat, S. Perruchas, C. Tard, and T. Gacoin, Appl. Phys. Lett. 89, 121118 (2006). 